\title{
Nonclassical Properties of a Class of Nonlinear Pair Coherent State
}

\author{
H. H. Salah \\ Department of Physics, Faculty of Science (Girls Branch), Al-Azher University, Nasr City, Cairo, Egypt
}

\begin{abstract}
In the present work a class of nonlinear pair coherent state introduced depending on the form of the nonlinearity function with examining some of its nonclassical properties. In particular, it is shown that such state exhibits nonclassical properties such as squeezing and sub-Poissonian behaviour. Also, the quasiprobability distribution functions (Wigner-function, Q-function) are discussed. Finally, the phase properties in Pegg-Barnett formalism is considered.
\end{abstract}

Keywords: Nonclassical state, Squeezing phenomenon, Quasi-distribution functions phase distribution

\section{Introduction}

The nonclassical states of the electromagnetic fields and of the atomic center-of mass motion for trapped ions are important in quantum optics due to their interesting characteristics. In recent years and according to the fundamental principles of quantum optics different types of nonclassical states have been designed [1-3]. Coherent states (CSs), their variants and generalizations have been extensively studied over the last four decades. [4, 5]. The usual CSs introduced by Glauber [6], are eigenstates of the annihilation operator $\hat{a}$ of the harmonic oscillator, i.e., $\hat{a}|\alpha\rangle=\alpha|\alpha\rangle$ and have widespread applications in various fields of physics (5, 7-9). There exist states of the electromagnetic field whose properties, like squeezing, higher order squeezing, antibunching and sub-Poissonian statistics $(10,11)$, are strictly quantum mechanical in nature.

On the other hand nonlinear coherent states (NLCS) or the f-coherent states are coherent states corresponding to nonlinear algebras. NLCS have been defined as the right eigenstate of a generalized annihilation operator $\hat{A}$. This is because in the case of nonlinear algebras the commutator $\left[\hat{A}, \hat{A}^{+}\right]$is nonlinear in the generators of the algebra [12-14]. The so-called nonlinear coherent states are defined as the right-hand eigenstates of the product of the boson annihilation operator $\hat{a}$ and a non-constant function of number operator $\hat{N}=\hat{a}^{+} \hat{a}$ [15], i.e., they satisfy

$$
f(\hat{N}) \hat{a}|\alpha, f\rangle=\alpha|\alpha, f\rangle,
$$

Where $f(\hat{N})$ is an operator-valued function of the number operator and $a$ is a complex eigenvalue. The ordinary coherent states $|\alpha\rangle$ are recovered for the special choice of $f(\hat{N})=1$. A class of NLCSs can be realized physically as the stationary states of the center-of-mass motion of a trapped ion [12]. The notion of the NLCS was generalized to the two-photon, one of the two-photon NLCS is the squeezed vacuum state and the other is squeezed first Fock state [16]. Previously, an exponential form of one-mode NLCS $|\alpha, f\rangle$ was given and proved that photon-added one-mode NLCSs are still NLCSs [17]. In analogy to the definition of the onemode NLCS, the two-mode nonlinear coherent state TMNLCS is defined as

$$
f\left(\hat{N}_{a}, \hat{N}_{b}\right) \hat{a} \hat{b}|\alpha, f, q\rangle=\alpha|\alpha, f, q\rangle,
$$

where $\hat{a}$ and $\hat{b}$ are boson annihilation operators; $f\left(\hat{N}_{a}, \hat{N}_{b}\right)$ is the function of the number operator $\hat{N}_{a}=\hat{a}^{+} \hat{a}$ and $\hat{N}_{b}=\hat{b}^{+} \hat{b} ; q$ is the photon number difference between two modes of the field. More recently a new kind of NLCSs using the inverse operators of the boson annihilation and creation operators and introduce a new kind of higher order squeezing and antibunching effect [18].

Pair coherent states (PCS) are regarded as an important type of correlated two-mode states, which possess prominent nonclassical properties. Such states denoted by $|\xi, q\rangle$ are eigenstates of the pair operator $(\hat{a} \hat{b})$ and 
the number difference $\left(\hat{n}_{a}-\hat{n}_{b}\right)$ where $\hat{a}$ and $\hat{b}$ are the annihilation operators of the field modes and $\hat{n}_{a}=\hat{a}^{+} \hat{a}$ and $\hat{n}_{b}=\hat{b}^{+} \hat{b}[19]$.These states satisfy

$$
\hat{a} \hat{b}|\xi, q\rangle=\xi|\xi, q\rangle \text { and }\left(\hat{n}_{a}-\hat{n}_{b}\right)|\xi, q\rangle=q|\xi, q\rangle \text {. }
$$

Experimentaly, its well known that the nonclassical PCS can be generated by considering the strong competition between the nonlinear gain (due to four-wave-mixing processes) and the nonlinear absorption in a two-photon medium by which photons are either created in pairs or destroyed in pairs [20,21]. Another scheme has been suggested for generating vibrational pair coherent states via the quantized motion of a trapped ion in a two-dimensional trap [22]. As an important extension of PCS in the finite dimensional Hilbert space, the finite dimensional PCS and the finite dimensional nonlinear PCS (NLPCS) have been introduced [23, 24]. Also new schemes for generating two new classes of the finite dimensional two-mode states of the vibrational motion of an ion in a two-dimensional trap have been demonstrated. Moreover, their some nonclassical properties have been investigated. The differences in phenomenon between two classes of finite-dimensional PCSs [23, 24], the standard PCSs [22] and NLPCSs [25] have also been found. Nonlinear extensions of the single-mode squeezed vacuum and squeezed coherent states are constructed. Nonlinear squeezed coherent states (NLSCSs) are defined and special cases of these states are discussed [26]. Also two-mode squeezed vacuum states are constructed and special cases of these states with their nonclassical properties are discussed [27]. On the basis of the concept of superposition principle in quantum optics, a new kind of finite-dimensional even and odd pair coherent states (EONPCSs) have been introduced with investigating their orthonormalized property, completeness relations and some nonclassical properties [28]. In a previous paper [29], we have introduce new even (odd) nonlinear pair coherent states EONLPCSs. In addition, some nonclassical features and quantum statistical properties of these new correlated two mode states have been investigated. Experimentally NLPCSs may also be generated in the vibrational motion of a trapped ion in a two-dimensional harmonic potential. So for some properties of the NLPCSs, it is possible to have some important applications in the fields of quantum optics and quantum information.

Therefore, the aim of the present paper is to introduce a class of nonlinear pair coherent states and then to study some of its non-classical properties. This manuscript is organized as follows, in section 2 we introduce the definition of NLPCSs. In section 3, we discuss some non-classical properties of NLPCSs, such as the squeezing phenomenon and the sub-poissonian distributions. In section 4, the quasi-probability distribution functions, more precisely we handle the Wigner and the Q-functions. Also the phase properties are considered, in section 5. Finally the conclusions are presented in section 6.

\section{Nonlinear Pair Coherent State (NLPCS)}

We define the NLPCS's $|\eta, q\rangle$ as the eigenstate of a generalized pair annihilation operator $\hat{A}=\hat{a} f_{a}\left(\hat{n}_{a}\right) \hat{b} f_{b}\left(\hat{n}_{b}\right)$ for the two modes, and the photon number difference between the two modes

$$
\begin{aligned}
& \hat{A}|\eta, q\rangle=\hat{a} f_{a}\left(\hat{n}_{a}\right) \hat{b} f_{b}\left(\hat{n}_{b}\right)|\eta, q\rangle=\eta|\eta, q\rangle, \\
& \left(\hat{a}^{+} \hat{a}-\hat{b}^{+} \hat{b}\right)|\eta, q\rangle=q|\eta, q\rangle
\end{aligned}
$$

Where $\eta$ is a complex variable (the state parameter) and $q$ parameter is an integer number. The $f_{i}\left(\hat{n}_{i}\right)$, (i=a, b), are well behaved operator valued functions of the operators $\hat{n}_{i}$, the NLPCS takes the form,

$$
|\eta, q\rangle=N(q) \sum_{n=0}^{\infty} \frac{|\eta|^{n}}{\sqrt{n !(n+q) ! f_{a}(n+q) ! f_{b}(n) !}}|n+q, n\rangle
$$

Obviously the inherent property of the nonlinear algebra depends on the choice of nonlinearity function $\mathrm{f}(\mathrm{n})$. However, prior to everything, one should determine the nonlinearity function of the nonlinear physical system of interest. We will consider the nonlinearity function of the centre-of-mass motion of a trapped ion which is the most popular system function in the nonlinear coherent state literature [30-32]. The nature of the nonlinearity depends on the choice of the function $\mathrm{f}(\mathrm{N})$ [13]. From equation (5), it is clear that for different choices of $\mathrm{f}(\mathrm{n})$ we shall get different NLSCS states. In the present study, we choose the nonlinearity function such as

In this case the state is given by,

$$
f_{i}\left(\hat{n}_{i}\right)=n_{i},(\mathrm{i}=\mathrm{a}, \mathrm{b})
$$




$$
|\eta, q\rangle=N(q) \sum_{n=0}^{\infty} \frac{|\eta|^{n}}{\sqrt{n !(n+q) !(n+q) ! n !}}|n+q, n\rangle
$$

In the Fock states of the two modes $\left|n_{a}, n_{b}\right\rangle$ and the normalization constant $N(q)$ is given by

$$
N(q)=\left[\sum_{n=0}^{\infty} \frac{|\eta|^{2 n}}{n !(n+q) !((n+q) ! n !)^{2}}\right]^{-1 / 2}
$$

The PCS can be obtained as a special case from equation (5) when $\mathrm{f}_{\mathrm{i}}(\mathrm{n}) !=1$.

\section{Non-classical properties}

To discuss the nonclassical properties of the present NLPCS state we shall consider two different phenomena. The first is the squeezing phenomenon which can be quantified via the quadrature variances for the normal squeezing case. As is well known, the squeezing means reduction in the noise of an optical signal below the vacuum limit, in addition to the possibility of potential applications in optical detection in communications networks of gravitational waves [33-38]. The second is Poissonian and sub-Poissonian behavior which can be measured using the Glauber second-order correlation function. Thus, in the following subsections we will investigate the influence of the controlling parameters q on the nonclassical behavior of the cavity field where, in particular, the squeezing phenomenon and the sub-Poissonian distribution are emphasized.

\subsection{The squeezing phenomenon}

Squeezing, a well-known non-classical effect, is a phenomenon in which variance in one of the quadrature components become less than that in vacuum state or coherent state [6] of radiation field at the cost of increased fluctuations in the other quadrature component. It is well known that squeezed light is a radiation field without a classical analogue where one of the quadratures of the electric field has less fluctuations than those for vacuum at the expense of increased fluctuations in the other quadrature. The usefulness of such light relates to several applications in optical communication networks [39], to interferometric techniques [36], and to optical waveguide trap [40]. Such systems can be constructed using linear optics, linear mixing of creation and annihilation operators, and nonlinear operations for state preparation and detection [41]. Mathematically the squeezing can be measured by calculating the Hermitian quadrature variances $\hat{X}$ and $\hat{Y}$. In the present subsection, the two-mode frequency sum squeezing defined by the quadrature operators $\hat{X}$ and $\hat{Y}$ [42] will be discussed. Therefore the variances of $\hat{X}$ and $\hat{Y}$ takes the form

$$
\hat{\mathrm{X}}=\frac{1}{2}\left(\hat{a} \hat{b}+\hat{a}^{+} \hat{b}^{+}\right), \quad \hat{\mathrm{Y}}=\frac{1}{2 i}\left(\hat{a} \hat{b}-\hat{a}^{+} \hat{b}^{+}\right),
$$

From which we have

$$
[\hat{\mathrm{X}}, \hat{\mathrm{Y}}]=i \hat{Z} \quad \text { with } \quad \hat{Z}=\frac{1}{2}\left(\hat{n}_{a}+\hat{n}_{b}+1\right),
$$

The variances, $\left\langle(\Delta \hat{\mathrm{X}})^{2}\right\rangle$ and $\left\langle(\Delta \hat{\mathrm{Y}})^{2}\right\rangle$, satisfy the Heisenberg uncertainty relation

$$
\left\langle(\Delta \hat{\mathrm{X}})^{2}\right\rangle\left\langle(\Delta \hat{\mathrm{Y}})^{2}\right\rangle \geq \frac{1}{4}\langle\hat{Z}\rangle^{2}
$$

Now we use the definition of the operators $\hat{X}$ and $\hat{Y}$ to calculate the quadrature variances $\left\langle(\Delta \hat{X})^{2}\right\rangle$ and $\left\langle(\Delta \hat{\mathrm{Y}})^{2}\right\rangle$

The variance is given in terms of annihilation and creation operators expectation values by

$$
\begin{aligned}
& \left\langle(\Delta \hat{X})^{2}\right\rangle=\frac{1}{4}+\frac{1}{4}\left[\left\langle\hat{n}_{a}+\hat{n}_{b}\right\rangle+2\left\langle\hat{n}_{a} \hat{n}_{b}\right\rangle+2 \Re e\left(\left\langle\hat{a}^{2} \hat{b}^{2}\right\rangle\right)\right]-(\mathfrak{R} e\langle(\hat{a} \hat{b})\rangle)^{2}, \\
& \left\langle(\Delta \hat{\mathrm{Y}})^{2}\right\rangle=\frac{1}{4}+\frac{1}{4}\left[\left\langle\hat{n}_{a}+\hat{n}_{b}\right\rangle+2\left\langle\hat{n}_{a} \hat{n}_{b}\right\rangle-2 \mathfrak{R} e\left(\left\langle\hat{a}^{2} \hat{b}^{2}\right\rangle\right)\right]+(\operatorname{Im}\langle(\hat{a} \hat{b})\rangle)^{2} .
\end{aligned}
$$

The state would possess $\hat{\mathrm{X}}$ or $\hat{\mathrm{Y}}$ quadrature frequency sum squeezing if the $S$-factor defined by 


$$
S_{X}(\eta)=\frac{2\left\langle(\Delta \hat{\mathrm{X}})^{2}\right\rangle-\langle\hat{Z}\rangle}{\langle\hat{Z}\rangle}, \text { or } \quad S_{Y}(\eta)=\frac{2\left\langle(\Delta \hat{Y})^{2}\right\rangle-\langle\hat{Z}\rangle}{\langle\hat{Z}\rangle}
$$

Satisfies the inequalities $-1 \leq S_{X}<0$ or $-1 \leq S_{Y}<0$.

In Fig. 1 the parameter $S(\eta)$ has been plotted against $\eta$ for different values of the parameter $q=1,2$, and 3 . According to this figure, it is visible that $S_{X} \prec 0$ for all range of $\eta$ and all $q$ - parameter. Thus one of the inequalities in (13) is satisfied. This implies that the NLPCS exhibits quadrature squeezing. Increasing the values of $q$ more squeezing can be seen. This means that the squeezing for the present state is too sensitive to any variation in the value of the parameter $q$. On the other hand, $S_{Y} \succ 0$ for all range of $\eta$ and different values of $q$, that means the NLPCS are never squeezed in Y quadratures. It will be very interesting to see if one can actually verify these predictions experimentally.

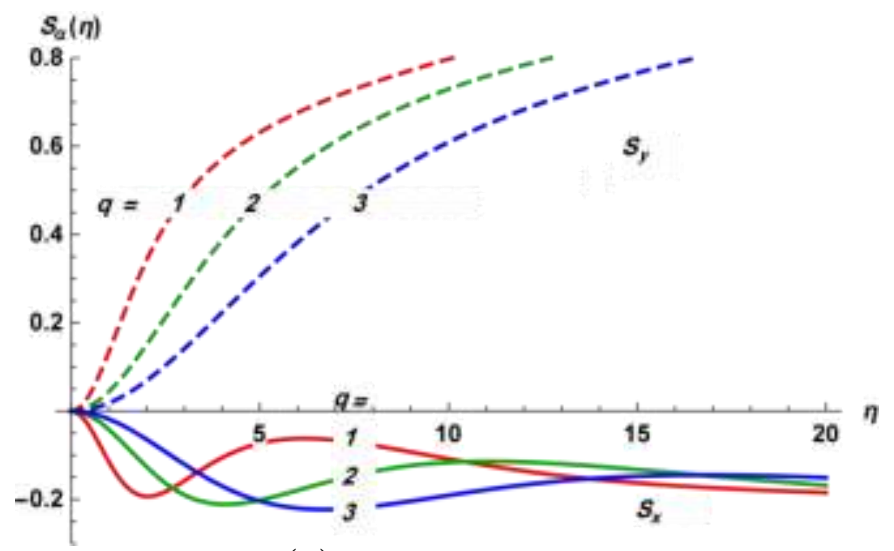

Fig. 1 The squeezing function $S(\eta)$ as a function of the parameter $\eta$ for $\mathrm{q}=1,2$ and 3 .

\subsection{Sub-Poissonian distributions}

Antibunching light is an example of nonclassical light and can be determined from a photocountingcorrelation measurement. Practicaly, the measurement can be performed by photon detectors based on photoelectric effect [23, 24]. Theoretically, the nonclassical behavior of the system can be done by examination of its second-order correlation function. In fact the correlation function is usually used to discuss the subPoissonian and super-Poissonian behavior of the photon distribution from which we can distinguish between the classical (bunching) and nonclassical (antibunching) behaviour of the system. The sub-Poissonian behavior is characterized by the fact that the variance of the photon number $\left\langle\left(\Delta \hat{n}_{i}\right)^{2}\right\rangle$ is less than the average mean photon number $\left\langle\hat{a}_{i}^{+} \hat{a}_{i}\right\rangle=\left\langle\hat{n}_{i}\right\rangle$. For the present state, this can be expressed by means of the normalized second-order correlation function for the mode $\mathrm{z}$ in a quantum state $|\eta, q\rangle[43]$ which defined as:

$$
g_{z}^{(2)}(\eta)=\frac{\left\langle\eta, q\left|\hat{n}_{z}\left(\hat{n}_{z}-1\right)\right| \eta, q\right\rangle}{\left\langle\eta, q\left|\hat{n}_{z}\right| \eta, q\right\rangle^{2}}, \forall z=a, b
$$

Where

$$
\left\langle\eta, q\left|\hat{n}_{z}\left(\hat{n}_{z}-1\right)\right| \eta, q\right\rangle=N^{2}(q) \sum_{n=0}^{\infty} \frac{|\eta|^{2 n}(n+z)(n+z-1)}{n !(n+q) !((n+q) ! n !)^{2}}
$$

and

$$
\left\langle\eta, q\left|\hat{n}_{z}\right| \eta, q\right\rangle=N^{2}(q) \sum_{n=0}^{\infty} \frac{|\eta|^{2 n}(n+z)}{n !(n+q) !((n+q) ! n !)^{2}}
$$

For the first mode $z=q$, while for the second mode $z=0$. 
The function $g_{z}^{(2)}(\eta)$ given by (14) for the mode $z$ serves as a measure of the deviation from the Poissonian distribution that corresponds to coherent states with $g_{z}^{(2)}(\eta)=1$. If $g_{z}^{(2)}(\eta)<1(>1)$, the distribution is called sub (super)-Poissonian, if $g_{z}^{(2)}(\eta)=2$ the distribution is called thermal and when $g_{z}^{(2)}(\eta)>2$ it is called super-thermal. To demonstrate these phenomena we have plotted the function against the parameter $\eta$. As shown in Fig. 2( a, b), the function $g_{z}^{(2)}(\eta)$ is positive and less than one for all values of $q$ and so the fully sub-Poissonian behavior of $|\eta, q\rangle$ is visible in all regions of $\eta$ for both modes. It is transparent that by increasing $q, g_{a}^{(2)}(\eta)$ for the mode $a$ get large values while $g_{b}^{(2)}(\eta)$ for the mode $b$ get small values, see Fig. 2 ( $\mathrm{a}, \mathrm{b}$ ) respectively.In other words, increasing $q$ results increasing depth of nonclassicality for the mode $b$. Generally it can be concluded that the NLPCS has sub-Poissonian nature for both modes.

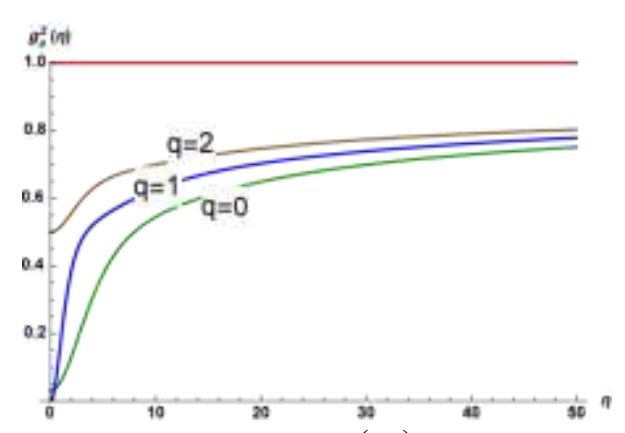

( a )

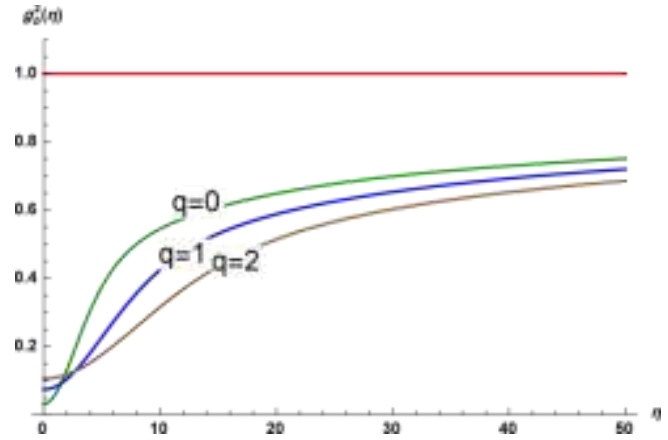

(b)

Fig. 2 The second-order correlation function $g_{z}^{(2)}(\eta)$ as a function of the parameter $\eta$ for $q=0,1$ and 2 ,

( a ) for mode $a$ and ( $\mathrm{b}$ ) for mode $b$

\section{Quasi-Probability Distribution Functions}

A convenient way of representing the states of the field is through quasiprobability distributions. They allow one to write down quantum expectation values of operators as integrals of the corresponding classical quantities weighted by the distribution function corresponding to the state. The quasiprobability distribution functions (QDFs) are perhaps one of the most well-known important functions in quantum optics [44 - 47]. For the statistical description of a microscopic system and to provide insight into the non-classical features of radiation fields, QDSs are needed to be studied [48-51]. There are three well known types of these functions: Glauber-Sudarshan P, Wigner-Moyal W, and Husimi-Kano Q functions corresponding to normally ordered, symmetric, and anti-normally ordered, respectively. W-function can take on negative values for some states and this is regarded as an indication of the non-classical effects. Also, it is well known that Q function is positive definite at any point in the phase for any quantum state. In fact these functions are important tools to give insight in the statistical description of a quantum mechanical system. Experimentally these functions can be measured via homodyne tomography [52]. In this section we shall concentrate on the $\mathrm{W}$ and $\mathrm{Q}$ functions only.

The $s$-parameterized characteristic function $(\mathrm{CF})$ for the two-mode states is defined as follows

$$
C\left(\lambda_{a}, \lambda_{b}, s\right)=\operatorname{Tr}\left[\hat{\rho} \hat{D}\left(\lambda_{a}\right) \hat{D}\left(\lambda_{b}\right)\right] \exp \left\{\frac{s}{2}\left(\left|\lambda_{a}\right|^{2}+\left|\lambda_{b}\right|^{2}\right)\right\}
$$

Where $\hat{\rho}$ is the density operator given by, $\hat{\rho}=|\eta, q\rangle\langle q, \eta|, \hat{D}(\lambda)$ is the displacement operator given by $\hat{D}(\lambda)=\exp \left(\lambda \hat{a}^{+}-\lambda^{*} \hat{a}\right)$, and $\lambda=|\lambda| e^{i \theta}$. Here, $s$ is ordering parameter where $s=(-1) 1$ means normal ordering and $s=0$ is symmetrical or Weyl ordering [53, 54].The s-parameterized QDF is the Fourier transformation of the $s$-parameterized CF $[53,55,56]$.

$$
F\left(\beta_{a}, \beta_{b}, s\right)=\left(\frac{1}{\pi^{2}}\right)^{2} \iint C\left(\lambda_{a}, \lambda_{b}, s\right) \exp \left(\lambda_{a}^{*} \beta_{a}+\lambda_{b}^{*} \beta_{b}-\lambda_{a} \beta_{a}^{*}-\lambda_{b} \beta_{b}^{*}\right) d^{2} \lambda_{a} d^{2} \lambda_{b} .
$$

Where the real parameter s defines the corresponding phase space distribution and associated with the ordering of the field bosonic operators. We consider a phase space QDF for the present states. To begin the state (7) will be written in the form 


$$
|\eta, q\rangle=\sum_{n=0}^{\infty} B_{n}(n, \eta, q)|n+q, n\rangle,
$$

Where

$$
B_{n}(n, \eta, q)=N(q) \frac{|\eta|^{n}}{\sqrt{n !(n+q) !(n+q) ! n !}}
$$

It is clear that, the probability of finding $(n+q)$ photons in the mode $a$, and $n$ photons in the mode $b$ in the state $|\eta, q\rangle$ is given by

$$
P(n+q, n)=\left|B_{n}(n, \eta, q)\right|^{2}
$$

After obtaining the parameterized characteristic function by using minor algebra and evaluating the integral in equation (18) for $s=0$ and $s=-1$, corresponding to the Wigner and the Q-function, respectively. Therefore the Wigner function and the Q-function, will be discussed in the next subsections.

\subsection{The Wigner function}

The Wigner function $W\left(\beta_{a}, \beta_{b}\right)$ can be obtained by inserting $s=0$ in equation (18) as follows

$$
\begin{aligned}
& W\left(\beta_{a}, \beta_{b}\right)=\frac{4}{\pi^{2}} \exp \left[-2\left(\left|\beta_{a}\right|^{2}+\left|\beta_{b}\right|^{2}\right)\right] \sum_{n=0}^{\infty} \sum_{l=0}^{n+q} \sum_{m=0}^{n}\left|B_{n}(n, \eta, q)\right|^{2}\left(\begin{array}{c}
n+q \\
l
\end{array}\right)\left(\begin{array}{c}
n \\
m
\end{array}\right) \\
& \times(-2)^{l+m} L_{l}\left[2\left|\beta_{a}\right|^{2}\right] L_{m}\left[2\left|\beta_{b}\right|^{2}\right]
\end{aligned}
$$

Where $L_{n}^{q}(x)$ are the associated Laguerre polynomials given by

$$
L_{n}^{q}(x)=\sum_{r=0}^{n}\left(\begin{array}{l}
n+q \\
n-r
\end{array}\right) \frac{(-1)^{r}}{r !} x^{r}
$$

To discuss the behavior of the Wigner function $W\left(\beta_{a}, \beta_{b}\right)$ we have plotted in Figure 3 the function against $x=\operatorname{Re}(\alpha)$ and $y=\operatorname{Im}(\alpha)$ for different values of the $q$-parameter $q=0,1,3$ and 8 with keeping $\eta$ parameter at constant value 0.5 , whereas $\beta_{a}$ and $\beta_{b}$ are taken to be equal. In the absence of the q-parameter, the Wigner function shows Gaussian distribution with one positive peak having wide base at the center. The peak has a symmetrical shape around zero, while its maximum reaches nearly the value 0.3 , see Fig. 3 (a). When the value of the $q$-parameter increases, for example $q=1$, we observe negative values attained by the Wigner function, which is a signature of the nonclassical effect. This is quite clear from Fig. 3 (b) where the peak changes its direction to downward.

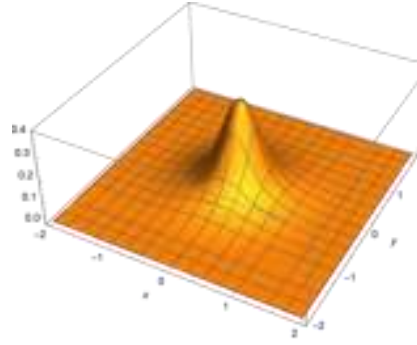

(a)

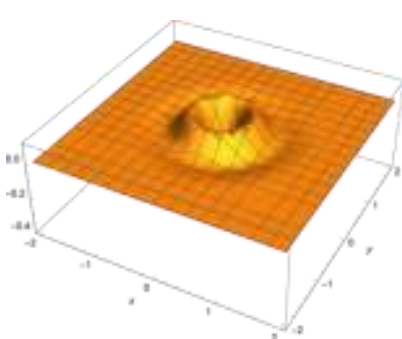

(c)

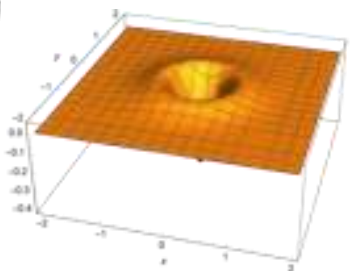

(b)

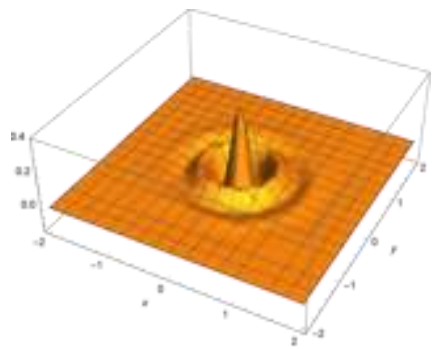

(d)

Fig. 3: The W-Wigner function against $(\operatorname{R}(\alpha))$ and $(\operatorname{Im}(\alpha))$ for fixed value of $\eta=0.5$, for NLPCS and (a ) $q=0$, (b ) $q=1$, (c) $q=3$ and (d ) $q=8$, respectively. 
For $q=3$ the Wigner function shows similar behavior to that of $q=1$, in addition dip negative values around the base are shown., see Fig.. 3 ( c ). Finally, for large values of the parameter $q=8$, the interference oscillatory with nonclassicality behavior starts to appear around the center of upward sharper positive peak. This indicates that the function gets more sensitive to the variation in the q-parameter and this of course reflects the change from Gaussian to non-Gaussian states; see Fig.3 (d).

\subsection{The Q-function}

The Husimi Q-function is a positive-definite at any point in the phase space for any quantum state, but its simple relation to antinormal operator products makes it difficult to interpret in terms of conventional photon counting or squeezing measurements [45]. By setting $s=1$ in equation (18), then the Q-function will has the form

$$
\begin{aligned}
& Q\left(\beta_{a}, \beta_{b}\right)=\frac{1}{\pi^{2}} \exp \left[-\left(\left|\beta_{a}\right|^{2}+\left|\beta_{b}\right|^{2}\right)\right] \sum_{n=0}^{\infty} \sum_{l=0}^{n+q} \sum_{m=0}^{n}\left|B_{n}(n, \eta, q)\right|^{2}\left(\begin{array}{c}
n+q \\
l
\end{array}\right)\left(\begin{array}{c}
n \\
m
\end{array}\right) \\
& \times(-1)^{l+m} L_{l}\left[\left|\beta_{a}\right|^{2}\right] L_{m}\left[\left|\beta_{b}\right|^{2}\right]
\end{aligned}
$$

Where $\beta_{a}, \beta_{b} \in C$, with $\left|\beta_{a}\right\rangle$ and $\left|\beta_{b}\right\rangle$ being the usual coherent states. Since there are four variables associated with the real and imaginary parts of $\beta_{a}$ and $\beta_{b}$.Therefore, by taking a subspace determined by, $\beta_{a}=\beta_{b}=\beta$ [42]. In this subspace the Q-function for the state (7) is expressed in the equivalent form

$$
Q(x, y)=\frac{1}{\pi^{2}} \exp \left[-2\left(x^{2}+y^{2}\right)\right]\left|\sum_{n=0}^{\infty} B_{n}(n, \eta, q) \frac{\beta^{2 n+q}}{\sqrt{n !(n+q) !}(n+q) ! n !}\right|^{2}
$$

Where $\beta=x+i y$.

The Q-function from equation.(25) for the NLPCS, has been plotted against $\operatorname{Re}(\beta)$ and $\operatorname{Im}(\beta)$ with the parameters having the values: $\eta=0.5$ and $q=0,1,2,3,5$ and 7 . It is clear from Fig. 4 ( a ) that in the absence of the q-parameter, the function exhibits Gaussian shape but with squeezing apparent on the contours of the base where it is elliptically shaped. At $q=1$, the Q- function exhibits two superimposed craters, as shown in Fig. 4 ( $\mathrm{b}$ ). With more increasing of $q$ the function keeps its shape but with increasing in its radius, spreading out and flattening in the phase space, Fig. 4 (c - f).

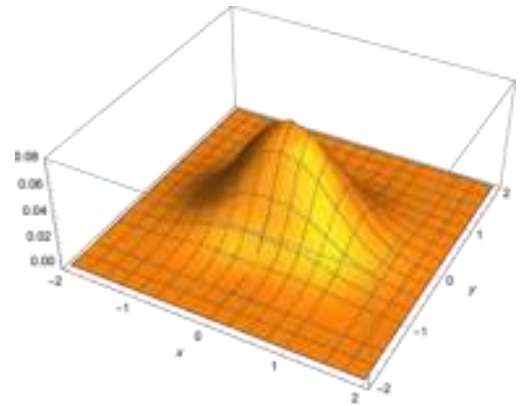

(a)

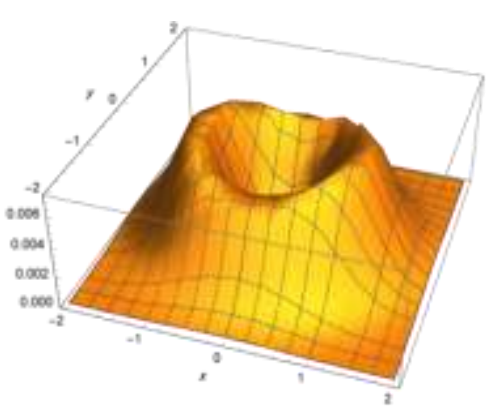

(c)

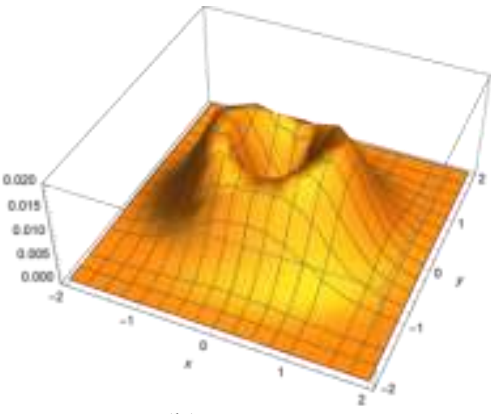

(b)

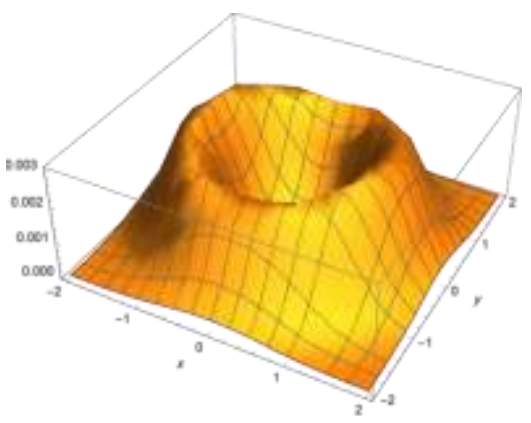

(d) 


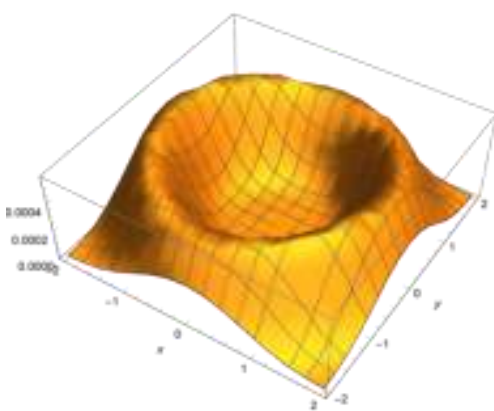

(e)

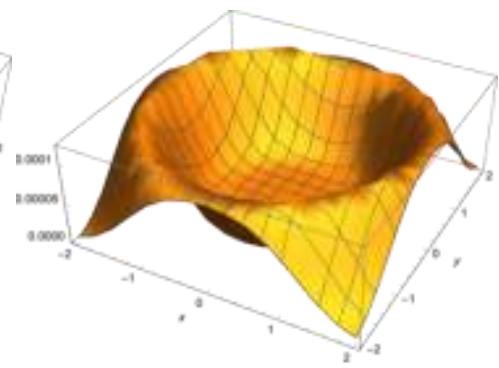

(f)

Fig. 4: $Q(x, y)$ as a function for NLPCS i.e. $s=-1$ with the parameters having the values: $\eta=0.5$ and different $q$ values.(a) $q=0$, (b) $q=1$, (c) $q=2$, (d ) $q=3$, (e ) $q=5$ and (f) $q=7$, respectively.

\section{Phase Distribution}

The notion of the phase in quantum optics has found renewed interest because of the existence of phase-dependent quantum noise. It is well known that the phase operator is defined as the projection operator on a particular phase state multiplied by the corresponding value of the phase. In this section, the phase properties using the Pegg-Barnett method [57, 58] are studied. Therefore the Pegg-Barnett phase distribution function $P_{P B}\left(\theta_{1}, \theta_{2}\right)$ for the present state will take the form [59]

$$
P_{P B}\left(\theta_{1}, \theta_{2}\right)=\frac{1}{4 \pi^{2}} \sum_{n, m=0}^{\infty} B_{n}(n, \eta, q) B_{m}^{*}(m, \eta, q) \exp \left\{i(n-m)\left(\theta_{1}+\theta_{2}\right)\right\}
$$

Where $\theta_{1}$ and $\theta_{2}$ are the phases related to the two modes $a$ and $b$ with the normalization condition

$$
\int_{-\pi}^{\pi} \int_{-\pi}^{\pi} P_{P B}\left(\theta_{1}, \theta_{2}\right) d \theta_{1} d \theta_{2}=1
$$

The phase distribution will depend on the sum of the phases of the two modes, due to the correlation between them. Therefore, we consider in equ. (26) $\theta=\theta_{1}+\theta_{2}$. The phase properties of NLPCS can be illustrated by plotting the Pegg-Barnett phase distribution function $P_{P B}(\theta)$ against the angle $\theta$ for different values of $\eta=1,10$ and 20 at two values of $q$ - parameter 1 and 5. The dependence of $P_{P B}(\theta)$ on $\eta$ is shown in Fig. ( 5 ). It is seen that as $\eta$ increases the distribution function $P_{P B}(\theta)$ becomes narrower. Worthwhile, increases of $q$ broadens the distribution $P_{P B}(\theta)$, which is seen from Fig. 5 ( b ). Also, the distribution function $P_{P B}(\theta)$ shows one-peak structure. This peak is centered around $\theta=0$ and the distribution is symmetric around the central peak. The maxima for the distribution at $\theta=0$ decrease by increasing $q$ for all values of $\eta$ , see Fig. 5 ( $a$ and $b$ ).
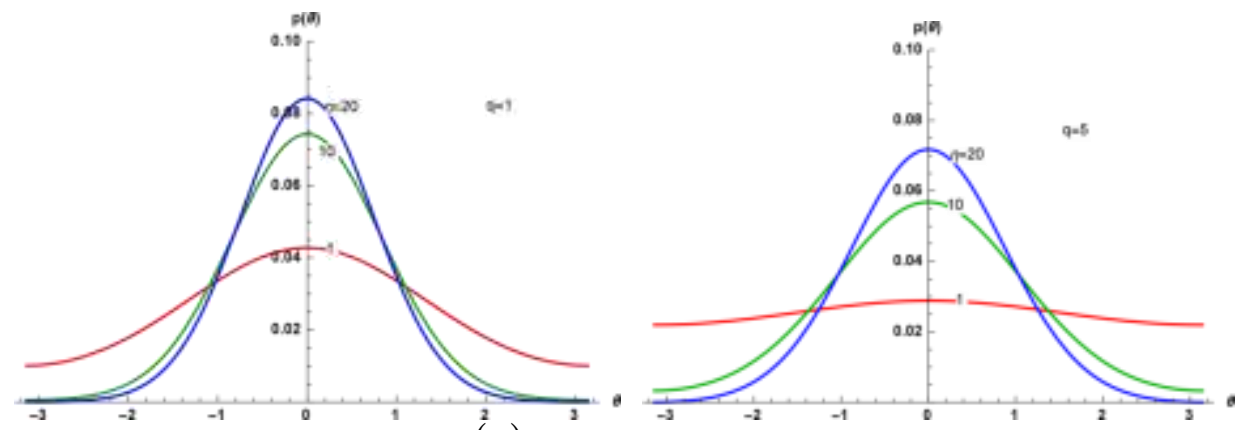

Fig. 5.The phase distribution function $P_{P B}(\theta)$ against the angle $\theta$ for different values of $\eta=1,10$ and 20 ,

( a ) $q=1$ and (b ) $q=5$.

\section{Conclusions}

We have studied the nonclassical behavior of the nonlinear pair coherent state for another form of the nonlinearity function. This class of state is characterized by two parameters: the q parameter which is related to 
the photon number difference in the two modes and $\eta$ the two-mode eigenvalue parameter. Properties of this state are investigated explicitly the squeezing phenomenon and sub-Poissonian distribution. Firstly, the NLPCS exhibits X-quadrature squeezing which is too sensitive to any variation in the value of the parameter $q$. Secondly, the state has fully sub-Poissonian nature for both modes. $a$ and $b$ in all ranges of $\eta$. Furthermore the quasiprobability distribution functions (the Wigner and Q-functions) has been discussed where the Wigner function gets more sensitive to the variation in the q-parameter and this of course reflects the change from Gaussian to non-Gaussian states. In other words, nonclassical signature of the NLPCS have been observed from negativity of the Wigner function. The Q-function exhibits Gaussian shape with squeezing apparent on the contours at $q=0$ and then with increasing of $q$ the function exhibits two superimposed craters with increasing in its radius, spreading out and flattening in the phase space. Finally, the phase distribution function $P_{P B}(\theta)$ shows one-peak structure with a symmetry around $\theta=0$. Therefore, the present state may be represents a good kind of two-mode state associated with PCS, and it may have some applications in the fields of quantum optics and quantum information.

\section{Acknowledgments}

The auther would like to thank Mrs. S. Sanad of the Mathemtics Department, Faculty of Science, Al-Azhar University (Girls Branch) for helpingin the programs for the figures.

\section{References}

[1]. S Mancini, Phys. Lett. A, 233, 291 (1997).

[2]. B Roy and P Roy, J. Opt. B: Quantum Semiclass. Opt., 2, 65; 505 (2000)

[3]. J S Wang, T K Liu, J Feng, J Z Sun and M S Zhan, Int. J. Theor. Phys. 42, 2855 (2003).

[4]. V.V. Dodonov, J. Opt. B: Quantum Semiclass. Opt. 4, R1 (2002);

[5]. J.R Klauder, B.S Skagerstam, Coherent States. Applications in Physics and Mathematical Physics; World Scientific, Singapore (1985).

[6]. [6] R. J. Glauber, Phys. Rev. 131, 2766 (1963).

[7]. A Perelomov, Generalized Coherent States and Their Applications; Springer, Berlin (1986).

[8]. W.M.Zhang, D.H.Feng, R.Gilmore, Rev. Mod. Phys., 62, 867-927 (1990).

[9]. S.T Ali, J.P Antoine, J.P.Gazeau, Coherent States, Wavelets and Their Generalizations; Springer, Berlin (2000).

[10]. D.F Walls, Nature, 306, 141-146 (1983).

[11]. R.Loudon, , P.L.Knight, J. Mod. Opt., 34, 709-759 (1987).

[12]. R.L.de Matos Filho and W. Vogel, Phys. Rev A54, 4560 (1996).

[13]. V.I. Manko, G.Marmo, E.C.G. Sudarshan and F. Zaccaria, Phys. Scrip 55,528 (1997).

[14]. A-S. F. Obada, M. Darwish and H. H. Salah, Journal of Modern Optics, Vol. 52, No. 9, 1263-1274 (2005).

[15]. L.C. Biedenharn, J. Phys. A: Math. Gen. 22, L873 (1989).

[16]. S.Sivakumar, Phys. Lett., A28, 257-262 (1998).

[17]. X.G. Wang, H.C. Fu, Mod. Phys. Lett. B ,13, 617 (1999).

[18]. Ji-Suo Wang, Xiang-Guo Meng, Bao-Long Liang \& Ke-Zhu Yan, Journal of Modern Optics, DOI:10. 1080/09500340. 2016. 1203464, 1-7 (2016).

[19]. E.M. Khalil, Int J Theor Phys, DOI 10.1007/s10773-007-9393-3 (2007).

[20]. G. S. Agarwal, Phys. Rev. Lett. 57, 827 (1986).

[21]. M.S. Malcuit, D.J. Gauthier, and R.W. Boyd, Phys. Rev. Lett. 55, 1086 (1985); R.W. Boyd, M.C. Malcuit, D.J. Gauthier, and K. Rzazewski, Phys. Rev. A 35, 1648 (1987).

[22]. S.C.Gou, , J.Steinbach, P.L.Knight, Phys. Rev. A 54, 4315 (1996).

[23]. A.-S.F. Obada and E.M. Khalil, Optics. Comm. 260, 19-24 (2006),

[24]. E.M. Khalil, J. Phys. A: Math. Gen. 39, 11053 (2006).

[25]. El-Orany, A A Faisal, and J Perina, Opt. Commun.197, 363 (2001).

[26]. A-S F Obada and G M Abd Al-Kader, J. Opt. B: Quantum Semiclass. Opt. 7 S635-S642 (2005).

[27]. G M Abd Al-Kader and A-S F Obada, Turk J Phys, 32,115-122 (2008).

[28]. Meng Xiang-Guo, Wang Ji-Suo, and Liu Tang-Kun, Chinese Physics B, Vol 17 No 9, 3350-3357 (2008).

[29]. H. H. Salah, IOSR Journal of Applied Physics, Volume 8, Issue 6, 113-122 (2016).

[30]. S Wallentowitz, and W. Vogel, Phys. Rev. A 55, 4438 (1997).

[31]. S Wallentowitz, and W. Vogel, Phys. Rev. A 58, 679 (1998).

[32]. Z. Kis, W. Vogel, and L. Davidovich, Phys. Rev. A 64, 033401 (2001).

[33]. J.H Shapiro, H.P Yuen, M.J.A Machado, IEEE Trans. Inf. Theory IT 25, 179 (1979).

[34]. M.J. Collett, C.W. Gardiner, Phys. Rev. A 30, 1386 (1984).

[35]. M.J. Collett, R. Loudon, J. Opt. Soc. Am. B 4, 1525 (1987).

[36]. C.M. Caves, B.L. Schumaker, Phys. Rev. A 31, 3068 (1985).

[37]. B.L. Schumaker, C.M. Caves, Phys. Rev. A 31, 3093 (1985).

[38]. C.M. Caves, Phys. Rev. D 23, 1693 (1981).

[39]. H.P Yuen and J.H Shapiro, IEEE Trans. Inform. Theory, 24, 657 (1978); 26, 78 (1980).

[40]. J. H Shapiro, Opt. Lett., 5, 351 (1980).

[41]. U Leonhardt, Measuring the Quantum State of Light (Cambridge: Cambridge University Press) (1997).

[42]. M Hillery, Phys. Rev. A, 40, 3147 (1989).

[43]. R. Loudon, the quantum theory of light; (Clarendon Press, Oxford) (1983).

[44]. W A Unsche, Acta Phys. Slov. 48, 385 (1998).

[45]. N. Moya-Cessa and P. L. Knight, Phys. Rev. A 48, 2479 (1993). 
[46]. T. h. Richter, J. Mod. Opt,. 48,1881 (2001).

[47]. W. A. Unsche, J. Opt. B: Quantum Semiclass. Opt. 6, 159 (2004).

[48]. E.Wigner, Phys. Rev. 40, 349 (1932).

[49]. E.Wigner, Z. Phys. Chem. B 19, 203 (1932)

[50]. K E Cahill and R J Glauber, Phys. Rev. 177, 1882 (1969).

[51]. M. Hillery, , R.F. O’Connell, , M.O. Scully, E.P.Winger, Phys. Rev. 106, 121 (1984).

[52]. B. Yurke, D. Stoler, Phys. Rev. Lett. 57, 13 (1986).

[53]. K E Cahill and R J Glauber, Phys. Rev. 177, 1857 (1969).

[54]. W Vogel and D-G Welsch, Quantum Optics; (Weinheim: Wiley-VCH) (2006)

[55]. S M Barnett and P M Radmore, Methods in Theoretical Quantum Optics; (Clarendon: OxfordUniversity Press) (1997).

[56]. W. P. Schleich, Quantum Optics in Phase Space ; (Wiley-Vch) (2001).

[57]. B. A Nguyen. and M. D Truong M. D., J. Opt. B.: Ouantum Semi. Opt., 4, 289 (2002).

[58]. D. T Pegg. and S. M Barnett., Eur. Phys. Lett., 6, 483 (1988); S. M Barnett. and D. T Pegg., J. Mod. Opt., 36, 7 (1989)

[59]. D. T Pegg. and S. M Barnett., Quantum Opt., 2, 225 (1997) 